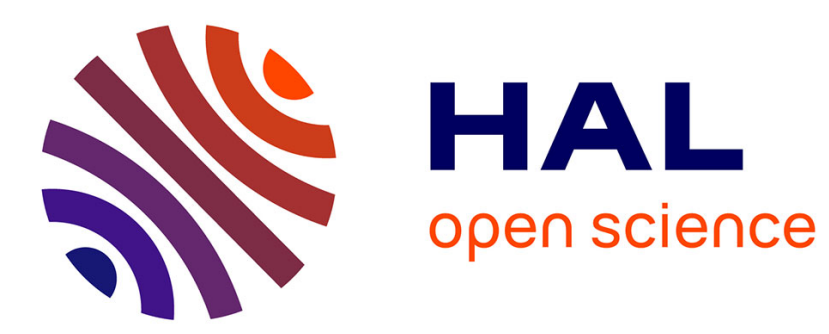

\title{
Non-Abelian geometric effect in quantum adiabatic transitions
}

Alain Joye, Charles-Edouard Pfister

\section{To cite this version:}

Alain Joye, Charles-Edouard Pfister. Non-Abelian geometric effect in quantum adiabatic transitions. Physical Review A : Atomic, molecular, and optical physics [1990-2015], 1993, 48 (4), 10.1103/PhysRevA.48.2598 . hal-01221147

\section{HAL Id: hal-01221147 https://hal.science/hal-01221147}

Submitted on 27 Oct 2015

HAL is a multi-disciplinary open access archive for the deposit and dissemination of scientific research documents, whether they are published or not. The documents may come from teaching and research institutions in France or abroad, or from public or private research centers.
L'archive ouverte pluridisciplinaire HAL, est destinée au dépôt et à la diffusion de documents scientifiques de niveau recherche, publiés ou non, émanant des établissements d'enseignement et de recherche français ou étrangers, des laboratoires publics ou privés. 


\title{
Non-Abelian geometric effect in quantum adiabatic transitions
}

\author{
A. Joye \\ Centre de Physique Théorique, CNRS Luminy Case 907, F-13288 Marseille, France \\ C.-E. Pfister \\ Département de Mathématiques, Ecole Polytechnique Fédérale de Lausanne, \\ CH-1015 Lausanne, Switzerland
}

(Received 9 November 1992)

\begin{abstract}
We establish a formula for the probability of a quantum adiabatic transition between two permanently degenerate energy levels which do not cross. This formula corresponds to the non-Abelian generalization of the Landau-Dykhne formula that is valid for the nondegenerate case. It applies in particular in cases of symmetry-induced degeneracy, a typical example being the Kramer degeneracy.
\end{abstract}

PACS number(s): 03.65. $-\mathrm{w}, 34.10 .+\mathrm{x}$

\section{INTRODUCTION}

For almost ten years now, the geometrical phase, or Berry phase, has been studied thoroughly from both the theoretical and experimental points of view. Many of these works can be found in the collection of reprints [1] or are reviewed in [2] and [3]. The starting point of all these developments was the famous paper [4]. (See also [5] concerning the geometrical interpretation of the results.) This geometrical phase was derived for timedependent systems whose Hamiltonians vary slowly in time, i.e., in the adiabatic limit. The adiabatic parameter $\varepsilon$ controlling this regime is defined as the inverse of the typical time scale of the system and the adiabatic limit is the limit $\varepsilon \rightarrow 0$. Soon after that Wilczek and Zee [6] generalized the case treated in [4] to what is sometimes called the "non-Abelian geometrical phase." The nonAbelian phase appears for systems displaying a permanent degeneracy, typically the Kramer degeneracy, and the paradigm model is a spin- $\frac{3}{2}$ in a quadrupole electric field [3]. This direction of research was pursued by several authors; see in particular [7-15].

Another important aspect of the adiabatic limit is the quantum adiabatic transition (QAT), often called the nonadiabatic transition, between two separate energy levels. This phenomenon is important in atomic and molecular physics - see, for example, [16-19] - and also in nuclear physics [20], in solid-state physics [21], or in laser physics $[22,23]$. Under an analyticity assumption on the time dependence of the Hamiltonian of the system and when the two energy levels display an avoided crossing, the probability of a QAT is given by the Landau-Zener formula [24-26]. See [27] and [28] for a mathematical analysis of the validity of this well-known formula. In more general situations this probability is usually computed with the Landau-Dykhne formula [29]. Recently it was realized simultaneously and independently by Berry [30] and Joye, Kunz, and Pfister [31] that this formula was not always valid and that a prefactor, of geometrical nature, was missing. This amplitude can be written as $\exp (2 \operatorname{Im} \theta)$ where $\theta$ is a complex phase which is the ana- lytic continuation of the geometrical phase in the complex time plane. This amplitude is related to the parallel transport of one eigenfunction along a loop around a particular complex level-crossing point. The probability of a QAT is then given by this geometrical factor times a decreasing exponential in $1 / \varepsilon$. The geometrical amplitude of the probability of a QAT has been measured in a spin experiment [32].

The main result of this paper is to show that in the presence of a permanent degeneracy of the energy levels it is possible to compute the asymptotic behavior of the probability of a QAT when $\varepsilon$ tends to zero. Our work generalizes the results of the papers [30] and [31] in the same way as the paper [6] generalizes [4]. The "nonAbelian geometrical amplitude" in our formula corresponds to the "non-Abelian geometrical factor" in [6] and is in fact obtained by an analytic continuation of it. The non-Abelian geometrical amplitude reflects properties of the parallel transport of a set of eigenvectors in the complex time plane around a complex crossing point of the two eigenvalues under consideration. We concentrate our exposition on the theoretical aspects for the simplest case. We expect that the effects predicted by the formula could be put into evidence experimentally (see [15]) in the same way as the geometrical amplitude of [30] and [31] has been measured by Zwanziger, Rucker, and Chingas [32], although such an experiment could be more delicate to perform. We think that mathematical rigor, whenever it is possible, is not the least important quality of a theoretical work. We therefore discuss carefully some mathematical aspects of the problem. Our analysis is presented in Secs. II and III. We first choose a basisindependent approach, in which the nature of the nonAbelian geometrical amplitude is quite evident. In order to treat concrete examples we come back to a basisdependent formulation of the results, which is the content of Theorem 3.2. In Sec. IV we study the paradigm model of a spin $\frac{3}{2}$ in a quadrupole electric field. We show in particular that we already have nontrivial geometrical effects in the case of real quadrupole Hamiltonians because these Hamiltonians depend on three real parameters. 
Several aspects of the QAT have been recently investigated in a series of papers [33-37]. Many of these developments can be extended to the present situation.

\section{THE GENERAL SITUATION}

Let $H(t)$ be an analytic time-dependent Hamiltonian with two distinct energy levels $e_{+}(t)$ and $e_{-}(t)$ such that the degeneracy of the energy levels is independent of $t$ and the degeneracy of $e_{+}(t)$ is the same as the degenera-

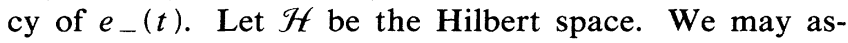
sume, without restricting the generality, that

$$
[H(t)]^{2}=\rho(t) \mathbb{1},
$$

with $\mathbb{1}$ the identity operator and $\rho(t)$ a strictly positive analytic function. The two energy levels are

$$
e_{ \pm}= \pm \sqrt{\rho(t)}, \quad \sqrt{1}=1
$$

and the corresponding spectral projections are

$$
P_{ \pm}(t)=\frac{1}{2}\left[1+\frac{H(t)}{e_{ \pm}(t)}\right) \text {. }
$$

We study scattering solutions of the Schrödinger equation

$$
i \varepsilon \frac{d}{d t} \psi_{\varepsilon}(t)=H(t) \psi_{\varepsilon}(t),
$$

where $1 / \varepsilon$ is the typical time scale of the system. These solutions are normalized, $\left\|\psi_{\varepsilon}(t)\right\|=1$, and are specified by a boundary condition at $t=-\infty$,

$$
\lim _{t \rightarrow-\infty}\left|\left\langle\chi-\mid \psi_{\varepsilon}(t)\right\rangle\right|=1
$$

where $\chi_{-}$is a unit vector in $P_{-}(-\infty) \mathcal{H}$ $\equiv \lim _{t \rightarrow-\infty} P_{-}(t) \mathcal{H}$. We want to find the asymptotic expression in $\varepsilon$ of the transition probability

$$
\mathcal{P}\left(\chi_{-}, \chi_{+} ; \varepsilon\right) \equiv \lim _{t \rightarrow+\infty}\left|\left\langle\chi_{+} \mid \psi_{\varepsilon}(t)\right\rangle\right|^{2}
$$

where $\chi_{+}$is a unit vector of $P_{+}(+\infty) \mathcal{H} \equiv$ $\lim _{t \rightarrow+\infty} P_{+}(t) \mathscr{H}$. The analysis of this probability is based on the existence of eigenvalue crossings at complex times. We state in the following condition assumptions which are valid throughout the paper.

Condition 1. The Hamiltonian has an analytic continuation $H(z)$ in a domain $\Omega$ of the complex plane, symmetrical with respect to the real axis. The set $X$ of zeros of $\rho$ in $\Omega$ has $2 m$ points, $m \geq 1$, and all zeros are simple. There exists $g>0$ such that

$$
\inf _{t \in \mathbb{R}} \rho(t) \geq g .
$$

There exist $\alpha>0$ and orthogonal projections $P_{ \pm}(+\infty), P_{ \pm}(-\infty)$ such that

$$
\lim _{t \rightarrow \pm \infty} \sup _{s}\left\|_{t+i s \in \Omega)}\right\| P_{ \pm}(t+i s)-P_{ \pm}( \pm \infty) \||t|^{1+\alpha}=0 .
$$

Remark. The zeros of $\rho$ occur in pairs $z$ and $\bar{z}$. The set
$X$ is therefore equal to $\left\{z_{j}, \overline{z_{j}}: j=1, \ldots, m\right\}$. By convention $\operatorname{Im} z_{j}>0$. Generically one pair of zeros is relevant for the dominant contribution to the asymptotic behavior of the transition probability. Which pair is actually relevant is discussed later on after the statement of Condition 2 in Sec. III. The other assumptions in Condition 1 are related to the noncrossing of the eigenvalues for real time and to the existence of limiting projections in order that the scattering problem be well posed.

Let $U_{\varepsilon}\left(t, t^{\prime}\right)$ be the evolution operator satisfying

$$
i \varepsilon \frac{d}{d t} U_{\varepsilon}\left(t, t^{\prime}\right)=H(t) U_{\varepsilon}\left(t, t^{\prime}\right), \quad U_{\varepsilon}\left(t^{\prime}, t^{\prime}\right)=1 \text {. }
$$

Then $\psi_{\varepsilon}(t)=U_{\varepsilon}\left(t, t^{\prime}\right) \psi_{\varepsilon}\left(t^{\prime}\right)$. The adiabatic theorem says that there exists another evolution $V_{\varepsilon}\left(t, t^{\prime}\right)$, the "adiabatic evolution," defined as the solution of the equation (see, e.g., [38])

$$
\begin{aligned}
i \varepsilon \frac{d}{d t} V_{\varepsilon}\left(t, t^{\prime}\right)=[H(t)+i \varepsilon & {\left[\frac{d}{d t}\left[P_{-}(t)\right] P_{-}(t)\right.} \\
& \left.\left.+\frac{d}{d t}\left[P_{+}(t)\right] P_{+}(t)\right]\right]
\end{aligned}
$$

$$
\times V_{\varepsilon}\left(t, t^{\prime}\right)
$$

$V_{\varepsilon}\left(t^{\prime}, t^{\prime}\right)=\mathbb{1}$

which is an approximate solution of (2.9):

$$
\sup _{\left(t_{1} \leq t \leq t_{2}\right)}\left\|U_{\varepsilon}\left(t, t^{\prime}\right)-V_{\varepsilon}\left(t, t^{\prime}\right)\right\| \leq O(\varepsilon) .
$$

In our case, because of assumption (2.8), the correction term $O(\varepsilon)$ is independent of the time interval $\left[t_{1}, t_{2}\right]$. Furthermore, we can write the adiabatic evolution as

$$
\begin{aligned}
V_{\varepsilon}\left(t, t^{\prime}\right)= & \exp \left[-\frac{i}{\varepsilon} \int_{t^{\prime}}^{t} e_{+}(s) d s\right] W\left(t, t^{\prime}\right) P_{+}\left(t^{\prime}\right) \\
& +\exp \left[-\frac{i}{\varepsilon} \int_{t^{\prime}}^{t} e_{-}(s) d s\right] W\left(t, t^{\prime}\right) P_{-}\left(t^{\prime}\right),
\end{aligned}
$$

where $W\left(t, t^{\prime}\right)$ is solution of the equation

$$
\begin{aligned}
& i \frac{d}{d t} W\left(t, t^{\prime}\right)=i\left[\frac{d}{d t}\left[P_{-}(t)\right] P_{-}(t)\right. \\
&\left.\quad+\frac{d}{d t}\left[P_{+}(t)\right] P_{+}(t)\right] W\left(t, t^{\prime}\right) \\
&=i\left(\frac{d}{d t} P_{-}(t), P_{-}(t)\right] W\left(t, t^{\prime}\right) \\
& \equiv K(t) W\left(t, t^{\prime}\right), \quad W\left(t^{\prime}, t^{\prime}\right)=\mathbb{1} .
\end{aligned}
$$

Both evolutions $V_{\varepsilon}\left(t, t^{\prime}\right)$ and $W\left(t, t^{\prime}\right)$ have the same intertwining property $[39,40]$, which reads for $W\left(t, t^{\prime}\right)$

$$
W\left(t, t^{\prime}\right) P_{ \pm}\left(t^{\prime}\right)=P_{ \pm}(t) W\left(t, t^{\prime}\right) .
$$


The adiabatic evolution is characterized by the intertwining property (2.14) and by (2.11). On the other hand, the evolution $W\left(t, t^{\prime}\right)$ depends only on the spectral projections. It specifies how eigenfunctions of $H\left(t^{\prime}\right)$ are transported into eigenfunctions of $H(t)$ during the adiabatic evolution, when we have factored out the dynamical phases. $W\left(t, t^{\prime}\right)$ has a purely geometrical interpretation in terms of parallel transport [4,5]. The characteristic property of this parallel transport is the following one. Let $\phi\left(t^{\prime}\right)$ be an eigenfunction of $H\left(t^{\prime}\right)$ for the eigenvalue $e_{ \pm}\left(t^{\prime}\right)$. The transported function $\phi(t)=W\left(t, t^{\prime}\right) \phi\left(t^{\prime}\right)$ is an eigenfunction of $H(t)$ for the eigenvalue $e_{ \pm}(t)$ such that

$$
P_{ \pm}(t) \frac{d}{d t} \phi(t)=0
$$

This property is a consequence of

$$
Q(t) \frac{d}{d t}[Q(t)] Q(t)=0,
$$

which is true for any projection $Q(t)$.

By Condition 1 the Hamiltonian is analytic in the domain $\Omega$, and therefore the evolution $U_{\varepsilon}$ has a singlevalued analytic continuation in this domain, which is given by the solution of the equation

$i \varepsilon \frac{d}{d z} U_{\varepsilon}\left(z, z^{\prime}\right)=H(z) U_{\varepsilon}\left(z, z^{\prime}\right), \quad U_{\varepsilon}\left(z^{\prime}, z^{\prime}\right)=\mathbb{1}$.

By contrast the generator $K$ of the evolution $W$ has a meromorphic continuation in the domain $\Omega$, with poles at the points of $X$, since

$$
\begin{aligned}
K(t) & =i\left(\frac{d}{d t} P_{-}(t), P_{-}(t)\right) \\
& =\frac{i}{4 \rho(t)}\left(\frac{d}{d t} H(t), H(t)\right) .
\end{aligned}
$$

Consequently $W$ has an analytic continuation in the punctured domain $\Omega \backslash X$, which is multivalued and which satisfies the equation

$$
i \frac{d}{d z} W\left(z, z^{\prime}\right)=K(z) W\left(z, z^{\prime}\right), \quad W\left(z^{\prime}, z^{\prime}\right)=\mathbb{1} .
$$

A similar assertion is true for $V_{\varepsilon}$.

Let us fix a reference time $t^{\prime}=0$. It is natural to express the evolution $U_{\varepsilon}(t, 0)$ as a product

$$
U_{\varepsilon}(t, 0) \equiv V_{\varepsilon}(t, 0) A_{\varepsilon}(t) .
$$

From (2.9) and (2.10) we derive a differential equation for $A_{\varepsilon}(t)$,

$$
\begin{array}{r}
i \frac{d}{d t} A_{\varepsilon}(t)=-V_{\varepsilon}(0, t) K(t) V_{\varepsilon}(t, 0) A_{\varepsilon}(t), \\
A_{\varepsilon}(0)=\mathbb{1} .
\end{array}
$$

$A_{\varepsilon}(t)$ is a unitary operator. Since $\|K(t)\|$ $=\left\|V_{\varepsilon}(0, t) K(t) V_{\varepsilon}(t, 0)\right\|$ is integrable on $\mathbb{R}$, the operators $\lim _{t \rightarrow \pm \infty} A_{\varepsilon}(t) \equiv A_{\varepsilon}( \pm \infty)$ exist, and we define the operator $A_{\varepsilon}(t,-\infty)$ by

$$
A_{\varepsilon}(t,-\infty) \equiv A_{\varepsilon}(t) A_{\varepsilon}^{-1}(-\infty) .
$$

This operator is the solution of the integral equation

$$
\begin{aligned}
A_{\varepsilon}(t,-\infty)=\mathbb{1}+i \int_{-\infty}^{t} & V_{\varepsilon}(0, s) K(s) \\
& \times V_{\varepsilon}(s, 0) A_{\varepsilon}(s,-\infty) d s .
\end{aligned}
$$

The next lemma gives an expression of the transition probability $\mathcal{P}\left(\chi_{-}, \chi_{+} ; \varepsilon\right)$ in terms of this operator.

Lemma 2.1. Let $\chi_{-} \in P_{-}(-\infty) \mathcal{H}$ and $\chi_{+} \in P_{+}(+\infty) \mathcal{H}$. The transition probability $\mathcal{P}\left(\chi_{-}, \chi_{+} ; \varepsilon\right)$ is given by

$$
\mathcal{P}\left(\chi_{-}, \chi_{+} ; \varepsilon\right)=\left|\left\langle\chi_{+} \mid W(+\infty, 0) P_{+}(0) A_{\varepsilon}(+\infty,-\infty) P_{-}(0) W(0,-\infty) \chi_{-}\right\rangle\right|^{2} .
$$

Remark. If we want to compute the transition $\mathcal{P}\left(\chi_{-}, \hat{\chi}_{-} ; \varepsilon\right)$ from $\chi_{-} \in P_{-}(-\infty) \mathcal{H}$ to a vector $\hat{\chi}_{-} \in P P_{-}(+\infty) \mathscr{H}$, we have a similar expression

$$
\mathcal{P}\left(\chi_{-}, \hat{\chi}_{-} ; \varepsilon\right)=\left|\left\langle\hat{\chi}_{-} \mid W(+\infty, 0) P_{-}(0) A_{\varepsilon}(+\infty,-\infty) P_{-}(0) W(0,-\infty) \chi_{-}\right\rangle\right|^{2} .
$$

This transition probability is of order 1 as $\varepsilon \rightarrow 0$, as is well known $[41,39]$.

Proof. By hypothesis $\psi_{\varepsilon}(t)$ satisfies the boundary condition $\lim _{t \rightarrow-\infty}\left|\left\langle\chi_{-} \mid \psi_{\varepsilon}(t)\right\rangle\right|=1$. Using (2.14) and $\psi_{\varepsilon}(t)=U_{\varepsilon}(t, 0) \psi_{\varepsilon}(0)$,

$$
\begin{aligned}
1= & \left.\lim _{t \rightarrow-\infty}\left|\chi_{-}\right| U_{\varepsilon}(t, 0) \psi_{\varepsilon}(0)\right\rangle \mid \\
= & \lim _{t \rightarrow-\infty}\left|\left\langle\chi_{-} \mid P_{-}(-\infty) V_{\varepsilon}(t, 0) A_{\varepsilon}(t) \psi_{\varepsilon}(0)\right\rangle\right| \\
= & \lim _{t \rightarrow-\infty} \mid\left\langle\chi_{-} \mid P_{-}(-\infty) P_{-}(t) \exp \left[-\frac{i}{\varepsilon} \int_{0}^{t} e_{-}(s) d s\right] W(t, 0) A_{\varepsilon}(t) \psi_{\varepsilon}(0)\right\rangle \\
& +\left\langle\chi_{-} \mid P_{-}(-\infty) P_{+}(t) \exp \left[-\frac{i}{\varepsilon} \int_{0}^{t} e_{+}(s) d s\right] W(t, 0) A_{\varepsilon}(t) \psi_{\varepsilon}(0)\right\rangle \mid \\
= & \left|\left\langle\chi_{-} \mid P_{-}(-\infty) W(-\infty, 0) A_{\varepsilon}(-\infty) \psi_{\varepsilon}(0)\right\rangle\right| \\
= & \left|\left\langle\chi_{-} \mid W(-\infty, 0) A_{\varepsilon}(-\infty) \psi_{\varepsilon}(0)\right\rangle\right|
\end{aligned}
$$


Thus the normalized vector $\varphi^{*}=W(-\infty, 0) A_{\varepsilon}(-\infty) \psi_{\varepsilon}(0) \in P_{-}(-\infty) \mathscr{H}$ differs from $\chi_{-}$by the phase factor $\left\langle\chi_{-} \mid \varphi^{*}\right\rangle$ only:

$$
\varphi^{*}=\left\langle\chi_{-} \mid \varphi^{*}\right\rangle \chi_{-} .
$$

Similarly, using (2.26),

$$
\begin{aligned}
\mathcal{P}\left(\chi_{-}, \chi_{+} ; \varepsilon\right) & =\lim _{t \rightarrow-\infty}\left|\left\langle\chi_{+} \mid U_{\varepsilon}(t, 0) \psi_{\varepsilon}(0)\right\rangle\right|^{2} \\
& =\left|\left\langle\chi_{+} \mid P_{+}(+\infty) W(+\infty, 0) A_{\varepsilon}(+\infty) \psi_{\varepsilon}(0)\right\rangle\right|^{2} \\
& =\left|\left\langle\chi_{+} \mid P_{+}(+\infty) W(+\infty, 0) A_{\varepsilon}(+\infty) \boldsymbol{A}_{\varepsilon}^{-1}(-\infty) W(0,-\infty) \varphi^{*}\right\rangle\right|^{2} \\
& =\left|\left\langle\chi_{+} \mid W(+\infty, 0) P_{+}(0) A_{\varepsilon}(+\infty,-\infty) P_{-}(0) W(0,-\infty) \chi_{-}\right\rangle\right|^{2} .
\end{aligned}
$$

By this lemma we only need to determine the operators $P_{+}(0) A_{\varepsilon}(+\infty,-\infty) P_{-}(0)$ or $P_{-}$(0) $A_{\varepsilon}(+\infty,-\infty) P_{-}(0)$. We therefore introduce the operators $C_{-}^{\varepsilon}(t)$ and $C_{+}^{\varepsilon}(t)$ which are defined as

$$
C_{-}^{\varepsilon}(t) \equiv P_{-}(0) A_{\varepsilon}(t,-\infty) P_{-}(0)
$$

and

$$
C_{+}^{\varepsilon}(t) \equiv P_{+}(0) A_{\varepsilon}(t,-\infty) P_{-}(0) .
$$

Let

$$
\widehat{K}(t) \equiv W(0, t) K(t) W(t, 0)
$$

and note that

$P_{-}(0) \hat{K}(t) P_{-}(0)=0, \quad P_{+}(0) \hat{K}(t) P_{+}(0)=0$.

From the integral equation (2.23) and (2.31) we have the following equations for the operators $C_{ \pm}^{\varepsilon}(t)$ :

$C_{-}^{\varepsilon}(t)=P_{-}(0)+i \int_{-\infty}^{t} \exp \left(\frac{i}{\varepsilon} \Delta(u)\right) \hat{K}(u) C_{+}^{\varepsilon}(u) d u$,

$C_{+}^{\varepsilon}(t)=i \int_{-\infty}^{t} \exp \left(-\frac{i}{\varepsilon} \Delta(u)\right) \widehat{K}(u) C_{-}^{\varepsilon}(u) d u$,

where $\Delta(u)=\int_{0}^{u}\left[e_{-}(s)-e_{+}(s)\right] d s$. It is also clear that the operators $C_{ \pm}^{\varepsilon}(t)$ have multivalued analytic continuations in the punctured domain $\Omega \backslash X$. The analytic continuations of $C_{ \pm}^{\varepsilon}$ along a path $\gamma$ are solutions of the above integral equations (2.32) along the path $\gamma$, where now $\Delta()$ and $\widehat{K}()$ denote the analytic continuations of these quantities along the path $\gamma$.

\section{TRANSITION PROBABILITY}

A basic idea, which is due to Landau [24] (see also [25]), is to use the multivaluedness of the eigenvalues and spectral projections in order to get two different expressions of the wave function $\psi_{\varepsilon}$. One writes the singlevalued function $\psi_{\varepsilon}$ in terms of the eigenvalues and eigenprojections. By considering analytic continuations of $\psi_{\varepsilon}$ along two nonhomotopic paths in the punctured domain one obtains two different expressions for $\psi_{\varepsilon}$. Let us im- plement this idea in our case. Let $z_{j} \in X$, and let $\gamma$ and $\hat{\gamma}$ be two paths from 0 to $z$ as in Fig. 1, so that the closed simple path $\hat{\gamma} \gamma^{-1}$ (first $\hat{\gamma}$ and then $\gamma^{-1}$ ) encircles only the crossing point $z_{j}$. Let $\eta$ be some path homotopic to $\hat{\gamma} \gamma^{-1}$ in the punctured domain. The value of the analytic continuation of the eigenvalues $e_{ \pm}(0)$ along the path $\eta$ when we come back to 0 is denoted by $e_{ \pm}(0 \mid \eta)$. Since the point $z_{j}$ is a simple zero of the function $\rho$, we have

$$
e_{ \pm}(0 \mid \eta)=e_{\mp}(0)
$$

and

$$
\int_{\widehat{\gamma}} e_{ \pm}(s) d s=\int_{\eta} e_{ \pm}(s) d s+\int_{\gamma} e_{\mp}(s) d s,
$$

where in all integrals the integrand is given by its analytic continuation along the integration path. Similarly we have (2.3)

$$
P_{ \pm}(0 \mid \eta)=P_{\mp}(0) .
$$

We can rewrite this result in terms of the operator $W$. As above, let $W(0 \mid \eta)$ be the operator which we get after analytic continuation of $W(, 0)$ along the path $\eta$. Since the intertwining property (2.14) is conserved by analytic continuation, we have

$W(0 \mid \eta) P_{ \pm}(0)=P_{ \pm}(0 \mid \eta) W(0 \mid \eta)=P_{\mp}(0) W(0 \mid \eta)$.

The operator $W(0 \mid \eta)$ gives the relation between the two determinations of the analytic continuation of $W$ along $\gamma$ and $\hat{\gamma}$,

$$
W(z \mid \hat{\gamma})=W(z \mid \gamma) W(0 \mid \eta) .
$$

The evolution $U_{\varepsilon}$ has a single-valued analytic continuation. Thus we can write

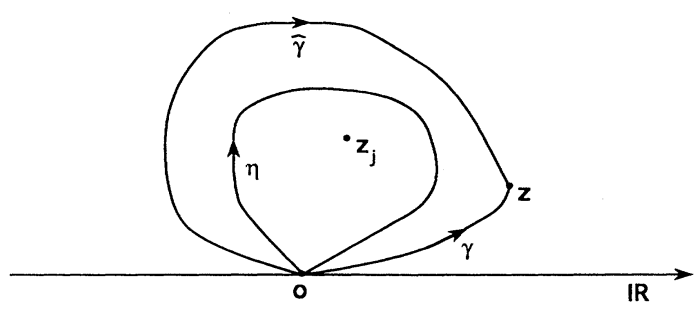

FIG. 1. The paths $\gamma, \hat{\gamma}$, and $\eta$. 


$$
\begin{aligned}
U_{\varepsilon}(z, 0)= & V_{\varepsilon}(z \mid \gamma) A_{\varepsilon}(z \mid \gamma) \\
= & V_{\varepsilon}(z \mid \hat{\gamma}) A_{\varepsilon}(z \mid \hat{\gamma}) \\
= & \exp \left[-\frac{i}{\varepsilon} \int_{\hat{\gamma}} e_{+}(s) d s\right) W(z \mid \hat{\gamma}) P_{+}(0) A_{\varepsilon}(z \mid \hat{\gamma})+\exp \left[-\frac{i}{\varepsilon} \int_{\hat{\gamma}} e_{-}(s) d s\right] W(z \mid \hat{\gamma}) P_{-}(0) A_{\varepsilon}(z \mid \hat{\gamma}) \\
= & \exp \left[-\frac{i}{\varepsilon} \int_{\eta} e_{+}(s) d s\right] \exp \left[-\frac{i}{\varepsilon} \int_{\gamma} e_{-}(s) d s\right] W(z \mid \gamma) P_{-}(0) W(0 \mid \eta) A_{\varepsilon}(z \mid \hat{\gamma}) \\
& +\exp \left[-\frac{i}{\varepsilon} \int_{\eta} e_{-}(s) d s\right] \exp \left[-\frac{i}{\varepsilon} \int_{\gamma} e_{+}(s) d s\right] W(z \mid \gamma) P_{+}(0) W(0 \mid \eta) A_{\varepsilon}(z \mid \hat{\gamma}) \\
= & \left.V_{\varepsilon}(z \mid \gamma)\left\{\exp \left[-\frac{i}{\varepsilon} \int e_{\eta}(s) d s\right] P_{-}(0)+\exp \int-\frac{i}{\varepsilon} \int_{\eta} e_{-}(s) d s\right] P_{+}(0)\right\} W(0 \mid \eta) A_{\varepsilon}(z \mid \hat{\gamma})
\end{aligned}
$$

Therefore

$$
A_{\varepsilon}(z \mid \gamma)=\left\{\exp \left[-\frac{i}{\varepsilon} \int_{\eta} e_{+}(s) d s\right] P_{-}(0)+\exp \left[-\frac{i}{\varepsilon} \int_{\eta} e_{-}(s) d s\right] P_{+}(0)\right\} W(0 \mid \eta) A_{\varepsilon}(z \mid \hat{\gamma}) .
$$

From that identity we immediately obtain

$$
C_{+}^{\varepsilon}(z \mid \gamma)=\exp \left[-\frac{i}{\varepsilon} \int_{\eta} e_{-}(s) d s\right] W(0 \mid \eta) C_{-}^{\varepsilon}(z \mid \hat{\gamma})
$$

and

$$
C_{-}^{\varepsilon}(z \mid \gamma)=\exp \left[-\frac{i}{\varepsilon} \int_{\eta} e_{+}(s) d s\right] W(0 \mid \eta) C_{+}^{\varepsilon}(z \mid \hat{\gamma}) .
$$

Let us suppose that we have a path $\hat{\gamma}$ in the punctured domain which goes from $-\infty$ to $+\infty$, passing over the point $z_{j}$ and no other point of $X$. Then we can use the above identities in order to express the transition probability $\mathcal{P}\left(\chi_{-}, \chi_{+} ; \varepsilon\right)$. Indeed, by Lemma 2.1

$$
\begin{aligned}
\mathcal{P}\left(\chi_{-}, \chi_{+} ; \varepsilon\right) & =\left|\left\langle\chi_{+} \mid W(+\infty, 0) C_{+}^{\varepsilon}(+\infty) W(0,-\infty) \chi_{-}\right\rangle\right|^{2} \\
& =\left.\exp \left[\frac{2}{\varepsilon} \operatorname{Im} \int_{\eta} e_{-}(s) d s\right]\left|\left\langle\chi_{+}\left|W(+\infty, 0) W(0 \mid \eta) C_{-}^{\varepsilon}(+\infty)\right| \hat{\gamma}\right) W(0,-\infty) \chi_{-}\right\rangle\right|^{2} .
\end{aligned}
$$

How do we select the eigenvalue crossing point $z_{j}$ ? This is indeed an important question, since in the above expression for the probability the exponential factor clearly depends on the choice of $z_{j}$. How do we control the operator $C_{-}^{\varepsilon}(+\infty \mid \hat{\gamma})$, which is given by the integral equations (2.32) integrated along the path $\hat{\gamma}$ ? The difficulty here is that $\Delta(u)$ is a complex quantity along the path $\hat{\gamma}$, and we want to take the limit $\varepsilon \rightarrow 0$. This point was solved in an important paper by Hwang and Pechukas [42]. They realized that one can control Eqs. (2.32) only along paths satisfying condition (d) below. It turns out that the two problems are closely related, as was pointed out in [31]. We can solve them if the following condition holds.

Condition 2. There exists a smooth path $\hat{\gamma}$ in the punctured domain $\Omega \backslash X$ and a point $z_{j} \in X$ with the following properties. Let $\hat{\gamma}$ be parametrized by $t \in \mathbb{R}$.

(a) $\lim _{t \rightarrow \pm \infty} \operatorname{Re} \hat{\gamma}(t)= \pm \infty$ and $\lim _{t \rightarrow \pm \infty} \operatorname{Im} \hat{\gamma}(t)=s_{ \pm}$.

(b) $\hat{\gamma}(t)$ goes above the point $z_{j} \in X, \operatorname{Im} \hat{\gamma}(t) \geq 0$.

(c) $\hat{\gamma}(t)$ goes below all points of $X \backslash\left\{z_{j}\right\}$.

(d) $\operatorname{Im} \Delta(\hat{\gamma}(t))$ is a nondecreasing function of $t$.
We call a path with property (d) a dissipative path. The real axis is a dissipative path, but in a very special way since $\operatorname{Im} \Delta \equiv 0$. Condition 2 is analyzed in the whole section 2.4 of [31], under the assumption that $\lim _{t \rightarrow \pm \infty} H(t)=H( \pm \infty)$ exist. In particular a geometrical interpretation of it is given. Under this extra assumption it is shown that points (a), (b), and (d) already imply point (c) of Condition 2 (see Theorem 2.1 in [31]). Moreover the relevant point $z_{j}$ is the closest point of $X$ to the real axis with respect to a metric naturally associated with the problem, which is different from the Euclidean metric. Examples illustrating this aspect are given in [31].

Lemma 3.1. If Condition 1 holds, then

$$
C_{-}^{\varepsilon}(+\infty)=P_{-}(0)+O(\varepsilon) .
$$

If furthermore Condition 2 holds, then

$$
\begin{aligned}
C_{+}^{\varepsilon}(+\infty)= & \exp \left[-\frac{i}{\varepsilon} \int_{\eta} e_{-}(z) d z\right] \\
& \times W(0 \mid \eta)\left[P_{-}(0)+O(\varepsilon)\right] .
\end{aligned}
$$


The path $\eta$ is as above (see Fig. 1).

Proof. The first statement follows by integrating (2.32) along the real axis, which is a dissipative path, and the second statement follows by considering the same integral equations along the dissipative path $\hat{\gamma}$. Using the method exposed in [31] for the nondegenerate case we can show exactly in the same manner that

$$
\sup _{t \in \mathbb{R}}\left\|C_{-}^{\varepsilon}[\hat{\gamma}(t)]-C_{-}^{\varepsilon}(-\infty)\right\|=O(\varepsilon) .
$$

The bound (3.11) corresponds to (2.57) of [31]. Therefore

$$
C_{-}^{\varepsilon}(+\infty \mid \hat{\gamma})=P_{-}(0)+O(\varepsilon) \text {. }
$$

$$
\begin{array}{ll}
\mathcal{P}\left(\chi_{-}, \chi_{+} ; \varepsilon\right)=\lim _{t \rightarrow+\infty}\left|\left\langle\chi_{+} \mid \psi_{\varepsilon}(t)\right\rangle\right|^{2}=\exp \left[\frac{2}{\varepsilon} \operatorname{Im} \int_{\eta} e_{-}(z) d z\right]\left\{\left|\left\langle\chi_{+} \mid W(+\infty, 0) W(0 \mid \eta) W(0,-\infty) \chi_{-}\right\rangle\right|^{2}+O(\varepsilon)\right\} . \\
\text { Similarly, if } \hat{\chi}_{-} \in P_{-}(+\infty) \mathcal{H}, \text { then } \\
P\left(\chi_{-}, \hat{\chi}_{-} ; \varepsilon\right)=\lim _{t \rightarrow+\infty}\left|\left\langle\hat{\chi}_{-} \mid \psi_{\varepsilon}(t)\right\rangle\right|^{2} & \begin{array}{l}
\text { where in (3.13) the integral is taken along a path from } \\
\text { along the same path. (This solution is well defined.) Of } \\
=\left|\left\langle\hat{\chi}_{-} \mid W(+\infty, 0) W(0,-\infty) \chi_{-}\right\rangle\right|^{2}+O(\varepsilon) .
\end{array} \\
\begin{array}{l}
\text { course expression (3.13) depends on the choice of the } \\
\text { path. If the chosen path is the real axis, then } \psi_{\text {ad }} \text { is an }
\end{array}
\end{array}
$$

The path $\eta$ is as above (see Fig. 1).

Comments.

(i) The relevant complex eigenvalue-crossing point $z_{j}$ is in the upper half-plane because we consider a QAT from the lowest energy level $e_{-}$to the highest energy level $e_{+}$. In the converse situation the relevant complex eigenvalue-crossing point is $\overline{z_{j}}$, and we must use instead of the path $\eta$ the complex conjugate of the path $\eta$.

(ii) Because of (2.8) we can define $W(t,-\infty)$, which gives the parallel transport of vectors of $P_{ \pm}(-\infty) \mathcal{H}$ into vectors of $P_{ \pm}(t) \mathcal{H}$. Thus in $\mathcal{P}\left(\chi_{-}, \hat{\chi}_{-} ; \varepsilon\right)$ the vector $W(+\infty, 0) W(0,-\infty) \chi_{-}=W(+\infty,-\infty) \chi_{-}$is the vector in $P_{-}(+\infty) \mathcal{H}$, which is obtained by transporting the vector $\chi_{-}$along the real axis. Similarly the vector $W(+\infty, 0) W(0 \mid \eta) W(0,-\infty) \chi_{\text {_ }}$ appearing in $\mathcal{P}\left(\chi_{-}, \chi_{+} ; \varepsilon\right)$ is obtained by transporting the vector $\chi_{-}$ along the real axis up to $t=0$, then along the path $\eta$, and finally along the real axis from $t=0$ to $+\infty$. However, since we follow the path $\eta$, which goes around the eigenvalue crossing $z_{j}$, the vector $W(+\infty, 0) W(0 \mid \eta) W(0,-\infty) \chi_{-} \in P_{+}(+\infty) \mathcal{H}$. Because of the uniformity of the condition (2.8) this vector is the same as the vector obtained by transporting the vector $\chi$ - along the path $\hat{\gamma}$.

(iii) Under the assumption that $\lim _{t \rightarrow \pm \infty} H(t)$ $=H( \pm \infty)$, the results of Theorem 3.1 can be illustrated in the following way. Ignoring the divergence of the real part of the integral in the next formula, we can write symbolically the adiabatic approximation at $z \in \Omega \backslash X$ as [see (2.12)]

$$
\begin{aligned}
\psi_{\mathrm{ad}}(z) & =V(z,-\infty) \chi_{-} \\
& =\exp \left[-\frac{i}{\varepsilon} \int_{-\infty}^{z} e_{-}\left(z^{\prime}\right) d z^{\prime}\right) W(z,-\infty) \chi_{-},
\end{aligned}
$$

Let us summarize these results in the next theorem.

Theorem 3.1. Let $H(t)$ be a Hamiltonian such that Conditions 1 and 2 hold. Let $\chi_{-} \in P_{-}(-\infty) \mathcal{H}$ and $\chi_{+} \in P_{+}(+\infty) \mathcal{H}$. If $\psi_{\varepsilon}(t)$ is a normalized solution of the Schrödinger equation

$$
i \varepsilon \frac{d}{d t} \psi_{\varepsilon}(t)=H(t) \psi_{\varepsilon}(t)
$$

subjected to the boundary condition

$$
\lim _{t \rightarrow-\infty}\left|\left\langle\chi_{-} \mid \psi_{\varepsilon}(t)\right\rangle\right|=1
$$

then

where in (3.13) the integral is taken along a path from $-\infty$ to $z$, and $W(z,-\infty)$ is the solution of Eq. (2.13) along the same path. (This solution is well defined.) Of course expression (3.13) depends on the choice of the path. If the chosen path is the real axis, then $\psi_{\text {ad }}$ is an approximate solution of the Schrödinger equation up to order $O(\varepsilon)$, and to the leading order

$$
\mathcal{P}\left(\chi_{-}, \hat{\chi}_{-} ; \varepsilon\right)=\lim _{t \rightarrow+\infty}\left|\left\langle\hat{\chi}_{-} \mid \psi_{\mathrm{ad}}(t)\right\rangle\right|^{2}
$$

On the other hand, if we choose the path $\hat{\gamma}$, then the function $\psi_{\mathrm{ad}}$ is not an approximate solution of the solution of the Schrödinger equation up to order $O(\varepsilon)$ along that path. It is very misleading to say that there is an adiabatic theorem in the complex plane along the path $\hat{\gamma}$, and that this theorem "says the same thing as its real axis counterpart" [42]. However, it is true that to the leading order

$$
\mathcal{P}\left(\chi_{-}, \chi_{+} ; \varepsilon\right)=\lim _{t \rightarrow+\infty}\left|\left\langle\chi_{+} \mid \psi_{\mathrm{ad}}(\hat{\gamma}(t))\right\rangle\right|^{2} .
$$

Note that in (3.14) and (3.15) the real part of the dynamical phase does not play any role, and in this sense the right-hand sides of these formulas are well defined.

(iv) The matrix generalizing the complex phase factor $\exp (-i \theta)$, which gives rise to the geometrical amplitude in the Landau-Dykhne formula, is

$$
P_{+}(0) W_{\eta}(0) P_{-}(0) \equiv M^{-} \text {. }
$$

Indeed

$$
\begin{aligned}
\left\langle\chi_{+} \mid W(+\infty, 0) W(0 \mid \eta) W(0,-\infty) \chi_{-}\right\rangle \\
=\left\langle\chi_{+}\right| P_{+}(+\infty) W(+\infty, 0) W(0 \mid \eta) W(0,-\infty) \\
\left.\quad \quad \times P_{-}(-\infty) \chi_{-}\right\rangle \\
=\left\langle\chi_{+} \mid W(+\infty, 0) M^{-} W(0,-\infty) \chi_{-}\right\rangle
\end{aligned}
$$

The operators $W(+\infty, 0)$ and $W(0,-\infty)$ are unitary since they transport vectors along the real axis. Thus we have to the leading order 


$$
\begin{aligned}
& \sup _{\left[\chi_{-} \in P_{-}(-\infty) \mathcal{H}^{\prime},\left\|\chi_{-}\right\|=1\right]} \mathcal{P}\left(\chi_{-}, \chi_{+} ; \varepsilon\right) \\
& {\left[\chi_{+} \in P_{+}(+\infty) \mathscr{H},\left\|\chi_{+}\right\|=1\right]} \\
& \quad=\left\|M^{-}\right\|^{2} \exp \left[\frac{2}{\varepsilon} \operatorname{Im} \int_{\eta} e_{-}(z) d z\right) .
\end{aligned}
$$

(v) If there exist limiting Hamiltonians such that $H(-\infty)=H(+\infty)$, then the operator

$$
P_{-}(-\infty) W(+\infty,-\infty) P_{-}(-\infty)
$$

appearing in

$$
\begin{aligned}
\mathscr{P}\left(\chi_{-}, \hat{\chi}_{-} ; \varepsilon\right) & \\
= & \left|\left\langle\hat{\chi}_{-} \mid P_{-}(-\infty) W(+\infty,-\infty) P_{-}(-\infty) \chi_{-}\right\rangle\right|^{2} \\
& +O(\varepsilon)
\end{aligned}
$$

is precisely the non-Abelian phase factor treated by Wilczek and Zee [6].

To end this section, we briefly indicate a basisdependent way of computing the parallel transport $W($ ) and the probability of a QAT. We suppose that the dimension of the Hilbert space $\mathcal{H}$ is $2 n$ so that each energy level is $n$-fold degenerate. Let $\varphi_{j}^{ \pm}(0), j=1,2, \ldots, n$, form an orthonormal basis of $P_{ \pm}(0) \mathcal{H}$. We set

$$
\varphi_{j}^{ \pm}(t)=W(t, 0) \varphi_{j}^{ \pm}(0) .
$$

By construction [see (2.14)]

$$
H(t) \varphi_{j}^{ \pm}(t)=e_{ \pm}(t) \varphi_{j}^{ \pm}(t), \quad j=1,2, \ldots, n
$$

and

$$
\left\langle\varphi_{j}^{ \pm}(t) \mid \varphi_{k}^{ \pm}(t)\right\rangle=\delta_{j k}, \quad \forall t \in \mathbb{R}
$$

These basis vectors are characterized by the property [see (2.15)]

$$
P_{ \pm}(t)\left[\varphi_{j}^{ \pm}(t)\right]^{\prime} \equiv 0, \quad \forall j=1,2, \ldots, n
$$

where we set ${ }^{\prime}=d / d t$. Consider now $\varphi_{j}^{ \pm}(0 \mid \eta)$, the analytic continuation of $\varphi_{j}^{ \pm}(0)$ along the loop $\eta$. By (3.4) we can write

$$
\varphi_{j}^{ \pm}(0 \mid \eta) \equiv W(0 \mid \eta) \varphi_{j}^{ \pm}(0)=\sum_{k=1}^{n} m_{k j}^{ \pm} \varphi_{k}^{\mp}(0) .
$$

This defines two $n \times n$ matrices $M^{ \pm}$whose elements are

$$
\left(M^{ \pm}\right)_{j k}=m_{j k}^{ \pm}, \quad j, k \in\{1,2, \ldots, n\}
$$

such that the operator $W(0 \mid \eta)$ in the basis

$$
\left\{\varphi_{1}^{-}(0), \varphi_{2}^{-}(0), \ldots, \varphi_{n}^{-}(0), \varphi_{1}^{+}(0), \varphi_{2}^{+}(0), \ldots, \varphi_{n}^{+}(0)\right\}
$$

is given by the block matrix [see (3.16)]

$$
W(0 \mid \eta)=\left[\begin{array}{cc}
0 & M^{+} \\
M^{-} & 0
\end{array}\right] \text {. }
$$

Note that the matrices $M^{ \pm}$satisfy the relation

$$
\operatorname{det}\left(M^{-}\right) \operatorname{det} M^{+}=(-1)^{n}
$$

as a direct consequence of the Liouville formula

$$
\begin{aligned}
\operatorname{det} W(t, 0) & =\exp \left\{\int_{0}^{t} \operatorname{tr}\left[P_{-}^{\prime}(u), P_{-}(u)\right] d u\right\} \operatorname{det} W(0,0) \\
& \equiv 1
\end{aligned}
$$

since $\operatorname{tr}\left[P_{-}^{\prime}(u), P_{-}(u)\right]=0$. Let $d_{j}^{ \pm}$be the coefficients of $\chi_{ \pm} \in P_{ \pm}( \pm \infty) \mathscr{H}$ in the basis vectors $\varphi_{j}^{ \pm}( \pm \infty)$ :

$$
d_{j}^{ \pm}=\left\langle\varphi_{j}^{ \pm}( \pm \infty) \mid \chi_{ \pm}\right\rangle, \quad j=1,2, \ldots, n .
$$

We can now express $\mathcal{P}\left(\chi_{-}, \chi_{+} ; \varepsilon\right)$ in a basis-dependent way.

Theorem 3.2. Under the hypotheses of Theorem 3.1

$$
\begin{aligned}
\mathcal{P}\left(\chi_{-}, \chi_{+} ; \varepsilon\right)= & \exp \left[\frac{2}{\varepsilon} \operatorname{Im} \int_{\eta} e_{-}(z) d z\right] \\
& \times\left\{\left|\sum_{j, k=1}^{n} \overline{d_{j}^{+}} m_{j k}^{-} d_{k}^{-}\right|^{2}+O(\varepsilon)\right\} \\
= & \exp \left[\frac{2}{\varepsilon} \operatorname{Im} \int_{\eta} e_{-}(z) d z\right] \\
& \times\left\{\left|\left(\mathbf{d}^{+} \mid M^{-} \mathbf{d}^{-}\right)\right|^{2}+O(\varepsilon)\right\},
\end{aligned}
$$

where $\left(\mathbf{d}^{ \pm}\right)_{j}=d_{j}^{ \pm}$and $(\mid)$is the scalar product in $\mathbb{C}^{n}$.

To compute the eigenvectors $W(t, 0) \varphi_{j}^{ \pm}(0)=\varphi_{j}^{ \pm}(t)$ we can proceed as follows if we know an orthonormal basis of instantaneous analytic eigenvectors of $H(t), \psi_{j}^{ \pm}(t)$, $j=1,2, \ldots, n$ :

$$
H(t) \psi_{j}^{ \pm}(t)=e_{ \pm}(t) \psi_{j}^{ \pm}(t), \quad j=1,2, \ldots, n .
$$

The eigenvectors $\varphi_{j}^{ \pm}(t)$ can be written as

$$
\varphi_{j}^{ \pm}(t)=\sum_{k=1}^{n} r_{k j}^{ \pm}(t) \psi_{k}^{ \pm}(t)
$$

with coefficients $r_{k j}^{ \pm}(t)$ to be determined. We choose $\varphi_{k}^{ \pm}(0)=\psi_{k}^{ \pm}(0)$. The vectors $\varphi_{k}^{ \pm}(t)$ being determined by the characteristic property (3.23), we can write

$$
\begin{aligned}
0 & \equiv\left\langle\psi_{k}^{ \pm}(t) \mid \varphi_{j}^{ \pm \prime}(t)\right\rangle \\
& =\sum_{l=1}^{n} r_{l j}^{ \pm \prime}(t) \delta_{l k}+r_{l j}^{ \pm}(t)\left\langle\psi_{k}^{ \pm}(t) \mid \psi_{l}^{ \pm \prime}(t)\right\rangle, \quad \forall j, k
\end{aligned}
$$

which yields the system of equations

$$
r_{k j}^{ \pm \prime}(t)=\sum_{l=1}^{n}-\left\langle\psi_{k}^{ \pm}(t) \mid \psi_{l}^{ \pm \prime}(t)\right\rangle r_{l j}^{ \pm}(t), \quad r_{k j}^{ \pm}(0)=\delta_{k j},
$$

or, in terms of the matrices

$$
\left[R^{ \pm}(t)\right]_{k j}=r_{k j}^{ \pm}(t)
$$

and

$$
\left[S^{ \pm}(t)\right]_{k j}=-\left\langle\psi_{k}^{ \pm}(t) \mid \psi_{j}^{ \pm \prime}(t)\right\rangle,
$$

we have the equations

$$
R^{ \pm \prime}(t)=S^{ \pm}(t) R^{ \pm}(t), \quad R^{ \pm}(0)=\mathbb{1}
$$

to be solved in $\mathbb{C}^{n}$, where 


$$
S^{ \pm}(t)^{*}=-S^{ \pm}(t) \quad t \in \mathbb{R}
$$

We have thus reduced the initial $2 n \times 2 n$ Schrödinger equation (2.4) to the $n \times n$ Schrödinger-like equation (3.37).

Note that we can always choose the phases of the vectors $\psi_{j}^{ \pm}(t)$ in such a way that $S^{ \pm}(t)$ is off diagonal. The matrices $S^{ \pm}(t)$ admit a multivalued analytic continuation whenever the vectors $\psi_{j}^{ \pm}(t)$ admit an analytic continuation, and the same is true for the solution of (3.37). Thus we can use the expansion (3.32) to compute $W(0 \mid \eta)$. Indeed, we can write the vectors $\psi_{j}^{ \pm}(t)$ under the form $\psi_{j}^{ \pm}(t)=V^{ \pm}(t) \psi_{j}^{ \pm}(0), t \in \mathbb{R}$, where $V^{ \pm}(t)$ are unitary $n \times n$ matrices defined by their elements $v_{k j}^{ \pm}(t)$ in the basis $\left\{\psi_{j}^{ \pm}(0)\right\}_{j=1}^{n}$ :

$$
v_{k j}^{ \pm}(t)=\left\langle\psi_{k}^{ \pm}(0) \mid \psi_{j}^{ \pm}(t)\right\rangle \text {. }
$$

These elements admit analytic continuations since the vectors $\psi_{j}^{ \pm}$do. Hence, for $t \in \mathbb{R}$,

$$
\left\langle\psi_{k}^{ \pm}(t) \mid \psi_{j}^{ \pm \prime}(t)\right\rangle=\left\langle\psi_{k}^{ \pm}(0) \mid V^{-1}(t) V^{ \pm \prime}(t) \psi_{j}^{ \pm}(0)\right\rangle
$$

admits an analytic extension. We remark, finally, that if the Hamiltonian is real, we can choose a real basis of instantaneous eigenvectors $\psi_{j}^{ \pm}(t)$ so that the generators $S^{ \pm}(t)$ are antisymmetric matrices on the real axis.

\section{QUADRUPOLE HAMILTONIAN}

We study in this last section a natural family of Hamiltonians displaying a permanent degeneracy. Let $Q=\left\{q_{i j}\right\}_{i, j=1,2,3}$ be a real symmetric $3 \times 3$ traceless matrix and $\left\{J_{i}\right\}_{i=1,2,3}$ be the components of a spin $j$. The quadrupole Hamiltonian

$$
H=\sum_{i, j=1}^{3} q_{i j} J_{i} J_{j}
$$

acting in the Hilbert space $\mathscr{H}=\mathbb{C}^{2 j+1}$ is the paradigm Hamiltonian for time-reversal systems, for which, in the case of half-integer values of $j$, the Kramer degeneracy occurs [3]. Let us briefly recall this notion.

Let $\tau=\exp \left(-i \pi J_{2}\right) C$ be the antiunitary time-reversal operator, where $C$ denotes complex conjugation. The operator $\tau$ is antiunitary:

$$
\langle\varphi \mid \tau \psi\rangle=\left\langle\psi \mid \tau^{\dagger} \varphi\right\rangle
$$

with

$$
\tau^{\dagger}=C \exp \left(i \pi J_{2}\right)=\tau^{-1}
$$

Using the standard representation of $\mathbf{J}$ where $J_{1}$ and $J_{3}$ are real and $J_{2}$ is pure imaginary, we find that $C$ commutes with $\exp \left(-i \pi J_{2}\right)$ and since $\exp \left(-i \pi J_{2}\right)$ represents a rotation of angle $\pi$ about the $y$ axis, we obtain

$$
\tau \mathbf{J}=-\mathbf{J} \tau
$$

Hence we immediately get

$$
[H, \tau]=0
$$

and

$$
\tau^{2}=\exp \left(-i 2 \pi J_{2}\right)= \begin{cases}+1, & j \in \mathbb{N} \\ -1, & j \in \frac{\mathbb{N}}{2}\end{cases}
$$

Let $j \in \mathbb{N} / 2$ and $\psi$ be such that

$$
H \psi=e \psi \text {. }
$$

Then

$$
H \tau \psi=e \tau \psi
$$

and

$$
\langle\psi \mid \tau \psi\rangle=-\left\langle\tau^{2} \psi \mid \tau \psi\right\rangle=-\langle\psi \mid \tau \psi\rangle,
$$

i.e.,

$$
\langle\psi \mid \tau \psi\rangle=0 \text {. }
$$

Thus any eigenvalue is at least twofold degenerate.

From now on we fix $j=\frac{3}{2}$, so that the dimension of the Hilbert space considered is 4 . As a consequence of this choice and of the Kramer degeneracy, either $H$ is a multiple of the identity or it has two distinct and twofolddegenerate eigenvalues. To see that the second alternative takes place, we must verify that $\operatorname{tr} H=0$. To derive this equality, we can make use of the tensorial properties of $H$ which imply that $\operatorname{tr} H$ is proportional to $\operatorname{tr} Q=0$ [14]. However, this identity will be clear from the explicit matrix representation of $H$ in the standard basis $\{|j, m\rangle\}_{-j \leq m \leq j}$, which we now give.

Using the relations

$$
\begin{aligned}
& J_{3}|j, m\rangle=m|j, m\rangle, \\
& \mathbf{J}^{2}|j, m\rangle=j(j+1)|j, m\rangle, \\
& J_{ \pm}|j, m\rangle=\sqrt{j(j+1)-m(m \pm 1)}|j, m \pm 1\rangle,
\end{aligned}
$$

where $J_{ \pm}=J_{1} \pm i J_{2}$ we easily obtain the matrices representing the operators $\left(J_{j} J_{i}+J_{i} J_{j}\right), \forall i, j=1,2,3$ in the standard basis ordered as follows:

$$
\left\{\left|\frac{3}{2},-\frac{3}{2}\right\rangle,\left|\frac{3}{2},-\frac{1}{2}\right\rangle,\left|\frac{3}{2}, \frac{1}{2}\right\rangle,\left|\frac{3}{2}, \frac{3}{2}\right\rangle\right\} \text {. }
$$

There are only five free coefficients in the matrix $Q$ which we choose to be

$$
\begin{aligned}
& B_{0}=\frac{\sqrt{3}}{2}\left(q_{11}-q_{22}\right), \\
& B_{1}=-\sqrt{3} q_{13} \\
& B_{2}=-\sqrt{3} q_{23} \\
& B_{3}=-\frac{3}{2}\left(q_{11}+q_{22}\right) \\
& B_{4}=\sqrt{3} q_{12} .
\end{aligned}
$$

We also define five corresponding operators, which take the block matrix forms in our basis: 


$$
\begin{aligned}
& L_{0}=\frac{J_{1}^{2}-J_{2}^{2}}{\sqrt{3}}=\left(\begin{array}{ll}
0 & 1 \\
\mathbb{1} & 0
\end{array}\right), \\
& L_{1}=-\frac{J_{1} J_{2}+J_{2} J_{1}}{\sqrt{3}}=\left[\begin{array}{cc}
\sigma_{1} & 0 \\
0 & -\sigma_{1}
\end{array}\right), \\
& L_{2}=-\frac{J_{2} J_{3}+J_{3} J_{2}}{\sqrt{3}}=\left[\begin{array}{cc}
\sigma_{2} & 0 \\
0 & -\sigma_{2}
\end{array}\right), \\
& L_{3}=\frac{1}{3}\left[2 J_{3}^{2}-\left(J_{1}^{2}+J_{2}^{2}\right)\right]=\left[\begin{array}{cc}
\sigma_{3} & 0 \\
0 & -\sigma_{3}
\end{array}\right], \\
& L_{4}=\frac{J_{1} J_{2}+J_{2} J_{1}}{\sqrt{3}}=\left(\begin{array}{cc}
0 & -i \mathbb{1} \\
i \mathbb{1} & 0
\end{array}\right],
\end{aligned}
$$

where $\sigma_{j}, j=1,2,3$, are the Pauli matrices.

Proposition 4.1. (i),

$$
\begin{aligned}
& L_{j}^{2}=\mathbb{1}, \quad \forall j=0,1, \ldots, 4 \\
& L_{j} L_{k}+L_{k} L_{j}=0, \quad \forall j \neq k \in\{0,1, \ldots, 4\} ;
\end{aligned}
$$

(ii),

$$
\begin{aligned}
& H=\sum_{i, j=1}^{3} q_{i j} J_{i} J_{j}=\sum_{j=0}^{4} B_{j} L_{j}, \\
& \operatorname{tr} H=0, \\
& H^{2}=\sum_{j=0}^{4} B_{j}^{2}=\frac{3}{2} \operatorname{tr}\left(Q^{2}\right) .
\end{aligned}
$$

The proof of that proposition causes no difficulty and will be omitted.

If we suppose that the matrix $Q(t)$ is chosen in such a way that the quadrupole Hamiltonian

$$
H(t)=\sum_{i, j=1}^{3} q_{i j}(t) J_{i} J_{j}=\sum_{j=0}^{4} B_{j}(t) L_{j}
$$

satisfies Condition 1, then we have in particular that

$$
\sum_{j=0}^{4} B_{j}^{2}(t) \geq g>0, \quad \forall t \in \mathbb{R} .
$$

The complex eigenvalue crossings are generically squareroot branching points of the eigenvalues

$$
e_{ \pm}(z)= \pm\left[\sum_{j=0}^{4} B_{j}^{2}(z)\right]^{1 / 2} \equiv \pm \lambda(z)
$$

In order to compute the operator $W(t, 0)$ we need a set of analytic eigenvectors $\psi_{j}^{ \pm}(t), j=1,2$, of our Hamiltonian

$$
\begin{aligned}
H(t) & =\left[\begin{array}{cc}
\mathbf{B}(t) \sigma & B_{0}(t)-i B_{4}(t) \\
B_{0}(t)+i B_{4}(t) & -\mathbf{B}(t) \sigma
\end{array}\right], \\
\mathbf{B}(t) & =\left[\begin{array}{l}
B_{1}(t) \\
B_{2}(t) \\
B_{3}(t)
\end{array}\right] .
\end{aligned}
$$

Let us adopt a block form for the vectors in $\mathbb{C}^{4}$ and write

$$
\chi=\left[\begin{array}{l}
\Phi \\
\Psi
\end{array}\right] \in \mathbb{C}^{4},
$$

where $\Phi, \Psi \in \mathbb{C}^{2}$. Defining $\langle\mid\rangle_{2}$ as the scalar product in $\mathbb{C}^{2}$, we have for any $\chi_{1}, \chi_{2} \in \mathbb{C}^{4}$

$$
\left\langle\chi_{1} \mid \chi_{2}\right\rangle=\left\langle\Phi_{1} \mid \Phi_{2}\right\rangle_{2}+\left\langle\Psi_{1} \mid \Psi_{2}\right\rangle_{2} \text {. }
$$

Consider the $2 \times 2$ matrix $(\mathbf{B} \sigma)$. Its eigenvalues are given by

$$
\pm \sqrt{B_{1}^{2}+B_{2}^{2}+B_{3}^{2}} \equiv \pm \mu
$$

and if $\left(B_{1}^{2}+B_{2}^{2}\right) \neq 0$ and $\mu \neq 0$, the vectors

$$
\zeta_{j}=\left(\begin{array}{c}
B_{3}+(-1)^{j} \mu \\
B_{1}+i B_{2}
\end{array}\right), \quad j=1,2
$$

satisfy

$$
(\mathbf{B} \sigma) \zeta_{j}=(-1)^{j} \mu \zeta_{j}, \quad j=1,2 .
$$

By means of these eigenvectors we can construct eigenvectors of $H$ as follows.

Lemma 4.1. Under the conditions $(\lambda+\mu)(\lambda-\mu)$ $=B_{0}^{2}+B_{4}^{2} \neq 0,\left(B_{1}^{2}+B_{2}^{2}\right) \neq 0$, and $\mu \neq 0$ the orthonormal vectors

$$
\begin{aligned}
\psi_{j}^{ \pm}= & {\left[\begin{array}{c}
\left(B_{0}-i B_{4}\right) \xi_{j} \\
{\left[ \pm \lambda-(-1)^{j} \mu\right] \xi_{j}}
\end{array}\right] } \\
& \times \frac{1}{2 \sqrt{\lambda \mu\left[\lambda \mp(-1)^{j} \mu\right]\left[\mu+(-1)^{j} B_{3}\right]}}
\end{aligned}
$$

satisfy

$$
H \psi_{j}^{ \pm}= \pm \lambda \psi_{j}^{ \pm}, \quad j=1,2 .
$$

Proof. Using the block form (4.18) of $H$, the property (4.23), and $\left\langle\zeta_{j} \mid \zeta_{k}\right\rangle_{2}=\delta_{j k} 2 \mu\left[\mu+(-1)^{k} B_{3}\right]$, the lemma is proven by explicit computation.

With this choice of eigenvectors, we can compute explicitly the coefficients of the generator $S^{ \pm}$in Eq. (3.37) for the Hamiltonian (4.15). After some straightforward algebraic manipulations we obtain the following.

Proposition 4.2. Let $\psi_{j}^{ \pm}(t)$ be the eigenvectors of $H(t)$ given in Lemma (4.1). Then, dropping the arguments,

$$
\begin{aligned}
\left\langle\psi_{j}^{ \pm} \mid \psi_{k}^{ \pm \prime}\right\rangle= & \frac{(-1)^{k}}{2 \mu^{2} \lambda}\left(\frac{B_{0}^{2}+B_{4}^{2}}{B_{1}^{2}+B_{2}^{2}}\right]^{1 / 2} \\
& \times\left[B_{1}\left(B_{3} B_{1}^{\prime}-B_{1} B_{3}^{\prime}\right)+B_{2}\left(B_{3} B_{2}^{\prime}-B_{2} B_{3}^{\prime}\right)\right] \\
& +\frac{i}{2 \mu \lambda}\left(\frac{B_{0}^{2}+B_{4}^{2}}{B_{1}^{2}+B_{2}^{2}}\right]^{1 / 2}\left(B_{1} B_{2}^{\prime}-B_{2} B_{1}^{\prime}\right), \quad j \neq k \\
\left\langle\psi_{j}^{ \pm} \mid \psi_{j}^{ \pm \prime}\right\rangle= & i\left[\frac{B_{4} B_{0}^{\prime}-B_{0} B_{4}^{\prime}}{2 \lambda\left[\lambda \mp(-1)^{j} \mu\right]}+\frac{B_{1} B_{2}^{\prime}-B_{2} B_{1}^{\prime}}{2 \mu\left[\mu+(-1)^{j} B_{3}\right]}\right] .
\end{aligned}
$$

These expressions are valid as long as the conditions of Lemma 4.1 are satisfied. By integration of Eq. (3.37) with the generator $S^{ \pm}(t)$ obtained from Proposition 4.2, we obtain the eigenvectors $\varphi_{j}^{ \pm}(t)$ (3.20) given by (3.32) and we can eventually compute the transition probability 
$\mathcal{P}\left(\chi_{-}, \chi_{+} ; \varepsilon\right)$ of Theorems 3.1 or 3.2 for any quadrupole Hamiltonian (4.15). In the general case, of course, the integration cannot be done analytically, but the analytic expressions derived above can be used to perform a numerical integration in any case.

However, there exists a family of quadrupole Hamiltonians for which the integration can be done analytically and for which the transition probability $\mathcal{P}\left(\chi_{-}, \chi_{+} ; \varepsilon\right)$ displays nontrivial properties. It is given by the threeparameter family of real quadrupole Hamiltonians characterized by

$$
q_{12}(t) \equiv q_{32}(t) \equiv 0 \text {, i.e., } B_{2}(t) \equiv B_{4}(t) \equiv 0 \text {. }
$$

Lemma 4.2. Let $H(t), t \in \mathbb{R}$ be a real quadrupole Hamiltonian satisfying Condition 1 . Then the solutions $R^{ \pm}(t)$ of (3.37) are given by

$$
R^{ \pm}(t)=\left(\begin{array}{cc}
\cos \theta(t) & -\sin \theta(t) \\
\sin \theta(t) & \cos \theta(t)
\end{array}\right)
$$

where

$\theta(t)=\int_{0}^{t} \frac{B_{0}(s)\left[B_{3}(s) B_{1}^{\prime}(s)-B_{1}(s) B_{3}^{\prime}(s)\right]}{2 \sqrt{B_{0}^{2}(s)+B_{1}^{2}(s)+B_{3}^{2}(s)\left[B_{1}^{2}(s)+B_{3}^{2}(s)\right]}} d s$.

Proof. By imposing the conditions, $B_{2}(t) \equiv B_{4}(t) \equiv 0$ we obtain from Proposition 4.2

$$
S^{ \pm}(t)=\left\langle\psi_{1}^{ \pm} \mid \psi_{2}^{ \pm \prime}\right\rangle(t)\left(\begin{array}{cc}
0 & -1 \\
1 & 0
\end{array}\right)
$$

with

$$
\begin{aligned}
\left\langle\psi_{1}^{ \pm} \mid \psi_{2}^{ \pm \prime}\right\rangle(t) & \\
& =\frac{B_{0}(t)\left[B_{3}(t) B_{1}^{\prime}(t)-B_{1}(t) B_{3}^{\prime}(t)\right]}{2 \sqrt{B_{0}^{2}(t)+B_{1}^{2}(t)+B_{3}^{2}(t)}\left[B_{1}^{2}(t)+B_{3}^{2}(t)\right]} .
\end{aligned}
$$

Hence

$$
\begin{aligned}
R^{ \pm}(t) & =\exp \left\{\theta(t)\left[\begin{array}{cc}
0 & -1 \\
1 & 0
\end{array}\right]\right\} \\
& =\left[\begin{array}{ll}
\cos \theta(t) & -\sin \theta(t) \\
\sin \theta(t) & \cos \theta(t)
\end{array}\right] .
\end{aligned}
$$

Note that the analytic continuation of $R^{ \pm}(t)$ along a path from 0 to $z$ is obtained directly from Lemma 4.2 provided the path does not pass through $X$ and $\left\{z \mid B_{1}^{2}(z)+B_{2}^{2}=0\right\}$.

Let us consider now the analytic continuation of the vectors $\psi_{j}^{ \pm}(t)$ along a loop $\eta$ based at the origin and encircling the eigenvalue crossing $z_{j}$. Let us denote by $f(0 \mid \eta)$ again the result of the analytic continuation of $f(0)$ along $\eta$, for any analytic function $f(z)$. Consider $\mu(z)=\sqrt{B_{1}^{2}(z)+B_{3}^{2}(z)}$. At $z_{j}$, the function $B_{0}^{2}(z)+B_{1}^{2}(z)+B_{3}^{2}(z)$ has a simple zero. If $B_{0}\left(z_{j}\right)=0$, then $B_{1}^{2}(z)+B_{3}^{2}(z)$ has a simple zero at $z_{j}$, and if $B_{0}\left(z_{j}\right) \neq 0$, then $B_{1}^{2}\left(z_{j}\right)+B_{3}^{2}\left(z_{j}\right) \neq 0$. Now, we choose $\eta$ in such a way that it encircles $z_{j}$ but no other zero of $\mu(z)$. Then,

$$
\mu(0 \mid \eta)= \begin{cases}+\mu(0) & \text { if } B_{0}\left(z_{j}\right) \neq 0 \\ -\mu(0) & \text { if } B_{0}\left(z_{j}\right)=0\end{cases}
$$

and by Condition 1

$$
\begin{aligned}
& B_{j}(0 \mid \eta)=B_{j}(0), \quad j=0, \ldots, 4 \\
& \lambda(0 \mid \eta)=-\lambda(0) .
\end{aligned}
$$

Thus

$$
\xi_{j}(0 \mid \eta)= \begin{cases}\zeta_{j}(0) & \text { if } B_{0}\left(z_{j}\right) \neq 0 \\ \zeta_{k}(0) & \text { if } B_{0}\left(z_{j}\right)=0\end{cases}
$$

and consequently

$$
\psi_{j}^{ \pm}(0 \mid \eta)=\omega_{j}^{ \pm} \times \begin{cases}\psi_{j}^{\mp}(0) & \text { if } B_{0}\left(z_{j}\right) \neq 0 \\ \psi_{k}^{\mp}(0) & \text { if } B_{0}\left(z_{j}\right)=0\end{cases}
$$

where $\omega_{j}^{ \pm}$equals +1 or -1 , depending on the total phase acquired along $\eta$ by the function under the square root in Lemma 4.1.

We have the following relations between the coefficients $\omega_{j}^{ \pm}$:

$$
\omega_{j}^{ \pm}= \begin{cases}-\omega_{k}^{ \pm} & \text {if } B_{0}\left(z_{j}\right) \neq 0 \\ +\omega_{k}^{ \pm} & \text {if } B_{0}\left(z_{j}\right)=0 .\end{cases}
$$

Indeed, consider the expression $\lambda^{2} \mu^{2}\left[\lambda \mp(-1)^{j} \mu\right]\left[\lambda \mp(-1)^{k} \mu\right]$

$$
\times\left[\mu+(-1)^{j} B_{3}\right]\left[\mu+(-1)^{k} B_{3}\right] .
$$

For $j \neq k$, it is equal to (remember that $B_{2} \equiv B_{4} \equiv 0$ )

$$
\lambda^{2} \mu^{2}\left(\lambda^{2}-\mu^{2}\right)\left(\mu^{2}-B_{3}^{2}\right)=\lambda^{2} \mu^{2} B_{0}^{2} B_{1}^{2} .
$$

This expression acquires a total phase along $\eta$ which is equal to

$$
\begin{aligned}
& m 4 \pi, \quad m \in \mathbf{Z} \text { if } B_{0}\left(z_{j}\right)=0 \\
& \pm 2 \pi+m 4 \pi, \quad m \in \mathbf{Z} \text { if } B_{0}\left(z_{j}\right) \neq 0 .
\end{aligned}
$$

As the phase of the analytic continuation of the square root of (4.33) along $\eta$ gives, by definition, the product $\omega_{j}^{ \pm} \omega_{k}^{ \pm}$, we obtain the relations (4.32). We recall the definition (3.32), which implies

$\varphi_{1}^{ \pm}(0 \mid \eta)=\cos [\theta(0 \mid \eta)] \psi_{1}^{ \pm}(0 \mid \eta)+\sin [\theta(0 \mid \eta)] \psi_{2}^{ \pm}(0 \mid \eta)$

$\varphi_{2}^{ \pm}(0 \mid \eta)=-\sin [\theta(0 \mid \eta)] \psi_{1}^{ \pm}(0 \mid \eta)+\cos [\theta(0 \mid \eta)] \psi_{2}^{ \pm}(0 \mid \eta)$,

and our choice $\varphi_{j}^{ \pm}(0)=\psi_{j}^{ \pm}(0)$. With (4.32), we obtain the following.

Proposition 4.3. Let $H(t)$ be a real quadrupole Hamiltonian satisfying Condition 1 . Then the matrices $M^{ \pm}$ defined by (3.24) are given by

$M^{ \pm}=\left\{\begin{array}{cc}\omega_{1}^{ \pm}\left[\begin{array}{cc}\cos [\theta(0 \mid \eta)] & -\sin [\theta(0 \mid \eta)] \\ -\sin [\theta(0 \mid \eta)] & -\cos [\theta(0 \mid \eta)]\end{array}\right] \text { if } B_{0}\left(z_{j}\right) \neq 0 \\ \omega_{1}^{ \pm}\left[\begin{array}{cc}\sin [\theta(0 \mid \eta)] & \cos [\theta(0 \mid \eta)] \\ \cos [\theta(0 \mid \eta)] & -\sin [\theta(0 \mid \eta)]\end{array}\right] \text { if } B_{0}\left(z_{j}\right)=0\end{array}\right.$

where 


$$
\theta(0 \mid \eta)=\int \frac{B_{0}(z)\left(B_{3}(z) B_{1}^{\prime}(z)-B_{1}(z) B_{3}^{\prime}(z)\right)}{\left.2 \sqrt{B_{0}^{2}(z)+B_{1}^{2}(z)+B_{3}^{2}(z)\left[B_{1}^{2}(z)\right.}+B_{3}^{2}(z)\right]} d z, \quad \omega_{1}^{ \pm}=1 \text { or }-1
$$

\section{Remarks.}

(i) The quantity $\theta(0 \mid \eta)$ has, in general, a nonvanishing imaginary part, so that $\sin \theta(0 \mid \eta)$ and $\cos \theta(0 \mid \eta)$ are not bounded by 1 .

(ii) As noted previously, the matrix $M^{-}$generalizes the geometric amplitude appearing when the energy levels are nondegenerate. In these situations, however, for real Hamiltonians, the geometrical amplitude has an absolute value of one (see [31]). This result is to be compared with the present situation, where, although our quadrupole Hamiltonian is real, $\operatorname{Im} \theta(0 \mid \eta) \neq 0$, so that $M^{ \pm}$are not unitary matrices. This qualitative difference of behavior comes from the fact that in the nondegenerate case, a real Hamiltonian is a two-parameter Hamiltonian, whereas in our case a real quadrupole Hamiltonian is a threeparameter Hamiltonian.

(iii) Finally, the formula expressing $\theta(0 \mid \eta)$ as a function of $B_{1}, B_{3}$, and $B_{0}$ has the same form as the formula yielding the complex phase factor in the general nondegenerate case which is a three-parameter family of Ham- iltonians [see (A.17) in [32]].

Lemma 4.1 together with Propositions 4.2 and 4.3 and Theorem 3.1 or 3.2 allow us to compute explicitly the transition probability $\mathcal{P}\left(\chi_{-}, \chi_{+} ; \varepsilon\right)$ from any vector $\chi_{-} \in P_{-}(-\infty) \mathbb{C}^{4}$ to any vector $\chi_{+} \in P_{+}(+\infty) \mathbb{C}^{4}$ when the Hamiltonian $H(t)$ is a real quadrupole Hamiltonian corresponding to a spin $j=\frac{3}{2}$. The leading term of the asymptotic expression of $\mathcal{P}\left(\chi_{-}, \chi_{+} ; \varepsilon\right)$ ranges from 0 to $\exp \left[(2 / \varepsilon) \operatorname{Im} \int_{\eta} e_{-}(z) d z\right]\left\|M^{-}\right\|^{2}$, depending on the vectors $\chi_{-}$and $\chi_{+}$. The norm $\left\|M^{-}\right\| \neq 1$, in general, so that this simple example displays all the characteristic features of $\mathcal{P}\left(\chi_{-}, \chi_{+} ; \varepsilon\right)$.

\section{ACKNOWLEDGMENTS}

This work has been partially supported by Fonds National Suisse de la Recherche, Grant No. 20-28521.90. A.J. thanks the Institut de Physique Theorique de l'EPFL, where a large part of this work has been performed, for its kind hospitality.
[1] A. Shapere and F. Wilczek, Geometric Phases in Physics (World Scientific, Singapore, 1989).

[2] J. W. Zwanziger, M. Koenig, and A. Pines, Annu. Rev. Phys. Chem. 41, 601 (1990).

[3] C. A. Mead, Rev. Mod. Phys. 64, 51 (1992).

[4] M. V. Berry, Proc. R. Soc. London Ser. A 392, 45 (1984).

[5] B. Simon, Phys. Rev. Lett. 51, 2167 (1983).

[6] F. Wilczek and A. Zee, Phys. Rev. Lett. 52, 2111 (1984).

[7] J. Moody, A. Shapere, and F. Wilczek, Phys. Rev. Lett. 56, 893 (1986).

[8] C. A. Mead, Phys. Rev. Lett. 59, 161 (1987).

[9] J. Segert, Phys. Rev. A 36, 10 (1987).

[10] J. Segert, J. Math. Phys. 28, 2102 (1987).

[11] R. Tycko, Phys. Rev. Lett. 58, 2281 (1987).

[12] A. Zee, Phys. Rev. A 38, 1 (1988).

[13] L. Sadun and J. Segert, J. Phys. A 22, L111 (1989).

[14] J. Avron, L. Sadun, J. Segert, and B. Simon, Commun. Math. Phys. 124, 595 (1989).

[15] J. W. Zwanziger, M. Koenig, and A. Pines, Phys. Rev. A 42, 3107 (1990).

[16] E. E. Nikitin and S. Ya. Umanskii, Theory of Slow Atomic Collisions (Springer-Verlag, Berlin, 1984).

[17] E. A. Solov'ev, Usp. Fiz. Nauk 157, 437 (1989) [Sov. Phys. Usp. 32, 228 (1989)].

[18] H. Nakamura, Int. Rev. Phys. Chem. 10, 123 (1990).

[19] S. G. Christov, Crit. Solid State Mater. Sci. 16, 337 (1990).

[20] A. Thiel, J. Phys. G 16, 867 (1990).

[21] M. Wilkinson, J. Phys. A 24, 2615 (1991).

[22] H. P. Breuer and M. Holthaus, Phys. Lett. A 140, 507 (1989).
[23] J. Z. Kaminski, Phys. Lett. A 151, 308 (1990).

[24] L. D. Landau, Collected Papers of L. D. Landau (Pergamon, Oxford, 1965).

[25] L. D. Landau and E. M. Lifshitz, Quantum Mechanics, 2nd ed. (Pergamon, Oxford, 1965), Sec. 53.

[26] C. Zener, Proc. R. Soc. London 137, 696 (1932).

[27] A. Joye and C.-E. Pfister, Phys. Lett. A 169, 62 (1992).

[28] A. Joye, Asymp. Anal. (to be published).

[29] A. M. Dykhne, Zh. Eksp. Teor. Fiz. 41, 1324 (1961) [Sov. Phys. JETP 14, 941 (1962)].

[30] M. V. Berry, Proc. R. Soc. London Ser. A 430, 405 (1990).

[31] A. Joye, H. Kunz, and C.-E. Pfister, Ann. Phys. (N.Y.) 208, 299 (1991).

[32] J. W. Zwanziger, S. P. Rucker, and G. C. Chingas, Phys. Rev. A 43, 3233 (1991).

[33] A. Joye and C.-E. Pfister, Commun. Math. Phys. 140, 15 (1991).

[34] A. Joye and C.-E. Pfister, J. Phys. A 24, 753 (1991).

[35] A. Joye, G. Mileti, and C.-E. Pfister, Phys. Rev. A 44, 4280 (1991).

[36] G. Nenciu, Commun. Math. Phys. 152, 479 (1993).

[37] A. Joye and C.-E. Pfister, J. Math. Phys. 34, 454 (1993).

[38] J. Avron, R. Seiler, and L. G. Yaffe, Commun. Math. Phys. 110, 33 (1987).

[39] T. Kato, J. Phys. Soc. Jpn. 5, 435 (1950).

[40] S. G. Krein, Linear Differential Equations in Banach Space (American Mathematical Society, Providence, RI, 1971).

[41] M. Born and V. Fock, Z. Phys. 51, 165 (1928).

[42] J. T. Hwang and P. Pechukas, J. Chem. Phys. 67, 4640 (1977). 